\title{
Biological Tissue Modeling with Agar Gel Phantom for Radiation Dosimetry of ${ }^{99 m} \mathrm{Tc}$
}

\author{
Liliana Aranda-Lara, Eugenio Torres-García, Rigoberto Oros-Pantoja \\ Laboratorio de Simulación Monte Carlo, Facultad de Medicina, Universidad Autónoma del Estado de México, \\ Toluca, México \\ Email: eugenio tg@yahoo.com.mx
}

Received 12 November 2013; revised 12 December 2013; accepted 19 December 2013

Copyright () 2014 by authors and Scientific Research Publishing Inc.

This work is licensed under the Creative Commons Attribution International License (CC BY).

http://creativecommons.org/licenses/by/4.0/

(c) (i) Open Access

\section{Abstract}

The biological tissue has been mimicked and replaced by other materials, which have shown certain radiological similarity determined by attenuation coefficient $(\mu)$, density and atomic number. Specifically, in molecular imaging and radiation therapy have been developed multifunctional radiopharmaceuticals which contain beta/gamma and/or light emitters to chronic degenerative diseases treatment. Therefore, it is necessary to develop phantoms that allow optical and radiometric characterization. Since the agar gel has shown to be a medium which allows to model biological tissue in phototherapy studies, the aim of this study is to determine whether the agar gel may be used as biological tissue substitutes in ${ }^{99 m}$ Tc dosimetry. Agar gel was prepared to $1 \%$ and $2.3 \%$ (water:agar) and its radiologicalproperties as: linear attenuation coefficient obtained by narrow beam geometry and XCOM software, density and effective atomic number $\left(Z_{\text {eff }}\right)$ were determined. Using the determined $\mu$, photontransmission was calculated by Monte Carlosimulation. The 99mTc source region was immersed in a water phantom, two source regions were used, one source region was filled with water and another with agar gel. For both cases; the cumulated activity $(\tilde{A})$ by conjugate view method, the absorbed doseper unitcumulated activity $(S)$ and absorbed dose $(D)$ were determined. The $2.3 \%$ concentration gel consistency facilitated its handling during a bigger irradiation time. A $\mu=0.151 \pm 0.021 / \mathrm{cm}$ was obtained and also this value was corroborated with the XCOM software. The agar gel density was $\rho=0.999 \pm 0.0004 \mathrm{~g} / \mathrm{cm}^{3}$ and $Z_{\text {eff }}=7.50$. The calculated cumulated activity presented $1 \%$ difference in both phantoms. The absorbed doseper unitcumulated activity was the same in both media, therefore the $D$ too. Agar gel showed to be equivalent to water in terms of radiological properties for $140 \mathrm{keV}$ photons, thus it can substitute soft tissue in ${ }^{99 m}$ Tc dosimetry.

\section{Keywords}

Agar Gel; ${ }^{99 m}$ Tc; Radiation Dosimetry; Tissue Substitute

How to cite this paper: Aranda-Lara, L., Torres-García, E. and Oros-Pantoja, R. (2014) Biological Tissue Modeling with Agar Gel Phantom for Radiation Dosimetry of ${ }^{99 m}$ Tc. Open Journal of Radiology, 4, 44-52. 


\section{Introduction}

In the field of the ionizing radiation dosimetry and medical physics the biological tissue has been mimicked and replaced by other materials, which have physical properties as those corresponding to the real tissue. The main purpose of tissue mimicking materials in dosimetry is to obtain an estimate of the absorbed dose as accurate as possible to human tissue. For example, water is considered as a reference material to perform dosimetric measurements because it has absorption and scattering properties of ionizing radiation similar to the soft tissue [1].

In general, based in its chemical composition, density and characteristics of interaction with photons and electrons different materials which can substitute biological tissue have been explored. The equivalence of tissue mimicking materials to water and/or biological tissue have been performed by Monte Carlo simulation and experimentally [2], [3].

The mass attenuation and mass energy absorption coefficients [4], [5], linear attenuation coefficient [6], [7] calculation of mass stopping and scattering [8]-[10], electron density and effective atomic number [5], [8], [11] of several materials have been used to determinate theradiological equivalence to water.

Equivalent tissue materials and Monte Carlo method have been used to perform dosimetric measurements in radiotherapy and nuclear medicine with the purpose of giving an accurate absorbed dose to patients and reduce it in critical tissues [6], [7], [12], [13]. Radiological properties and water equivalence of hydrogels have been previously studied to be used in x-ray, electron beams and brachytherapy sources dosimetry [4], [8], [10] due to these materials allow to know the three dimensional distribution of absorbed dose.

In nuclear medicine, the technetium-99m is the most commonly used radioisotope in diagnostic procedures. Its "short" physical half-life of 6 hours and its gamma ray semission $(140 \mathrm{keV})$ allows adequate image quality for correct diagnosis. These features permit its application in the evaluation of equivalent materials to water or tissue with the purpose of developing solid phantoms [6].

Moreover, hydrogels are also used in bio-optical research to elaborate phantoms with similar characteristics to soft tissue and reproduce the interaction of visible light and infrared radiation that occurs in the biological tissue [14]. Furthermore, they enable to construct semisolid objects with specific geometry and add inorganic substances which act as scattering or attenuation agents [15].

Agar is a polysaccharide complex which has the capacity to hold water within its structure due to the presence of hydrophilic group such as: $\mathrm{OH},-\mathrm{COOH},-\mathrm{CONH}_{2}, \mathrm{y}-\mathrm{SO}_{3} \mathrm{H}$ [16], [17]. The agar gel phantom shows absorption and scattering characteristic similar to soft tissue and it is used to measure heat production and its distribution when the medium (with and without gold nanoparticles) is irradiated by laser beam [15], [18], [19].

Nowadays in molecular imaging and targeted radiotherapy are developing of novel multimodality imaging agents containing beta/gamma and/or light emitters in its structure, whose mechanism of action is cancer cell killing by biological processes (angiogenesis inhibition, apoptosis induction), targeted radiotherapy and photothermal therapy [20], [21]. Thus, it is necessary to develop phantoms that allow the radiometric and optical characterization of these imaging agents. It has been shown that the agar gel under specific conditions of preparation mimics the biological tissue for optical characterization, therefore the aim of this study was to determinate if the agar gel can be used with radiometric purpose as soft biological tissue substitute when it is irradiated with $140 \mathrm{keV}$ photons.

\section{Materials and Methods}

The molecular formula of agar powder $\left(\mathrm{C}_{12} \mathrm{H}_{18} \mathrm{O}_{9}\right)_{n}$ was obtained from manufacturer [22] and used to calculate its elemental weight fractions of agar and water in the gel sample. The agar powder was dissolved in water at 1 and 2.3\% (water:agar), the mixture was heated up to the boiling point during 2 minutes with constant stirring and was gelled at room temperature. The agar gel phantom was obtained a cylindrical blocks of 5, 10, 15, 20, 25 $\mathrm{cm}$ thick and $2.4 \mathrm{~cm}$ radius.

The $140 \mathrm{keV}$ photon beam was obtained from a source of ${ }^{99 \mathrm{~m}} \mathrm{Tc}$ with approximate activity of $11.1 \mathrm{MBq}$, it was placed within a lead container to collimate and modify the output beam diameter to generate three photon fluencies. The primary photons transmission is determined by narrow beam geometry [23] with source-detector distance of $1 \mathrm{~m}$, as is shows in Figure 1.

Transmission scans were performed on a dual head gamma camera (Siemens, e.cam) equipped with low energy collimator and the electronic collimation was realized with a 15\% energy window centered in $140 \mathrm{keV}$. During 5 


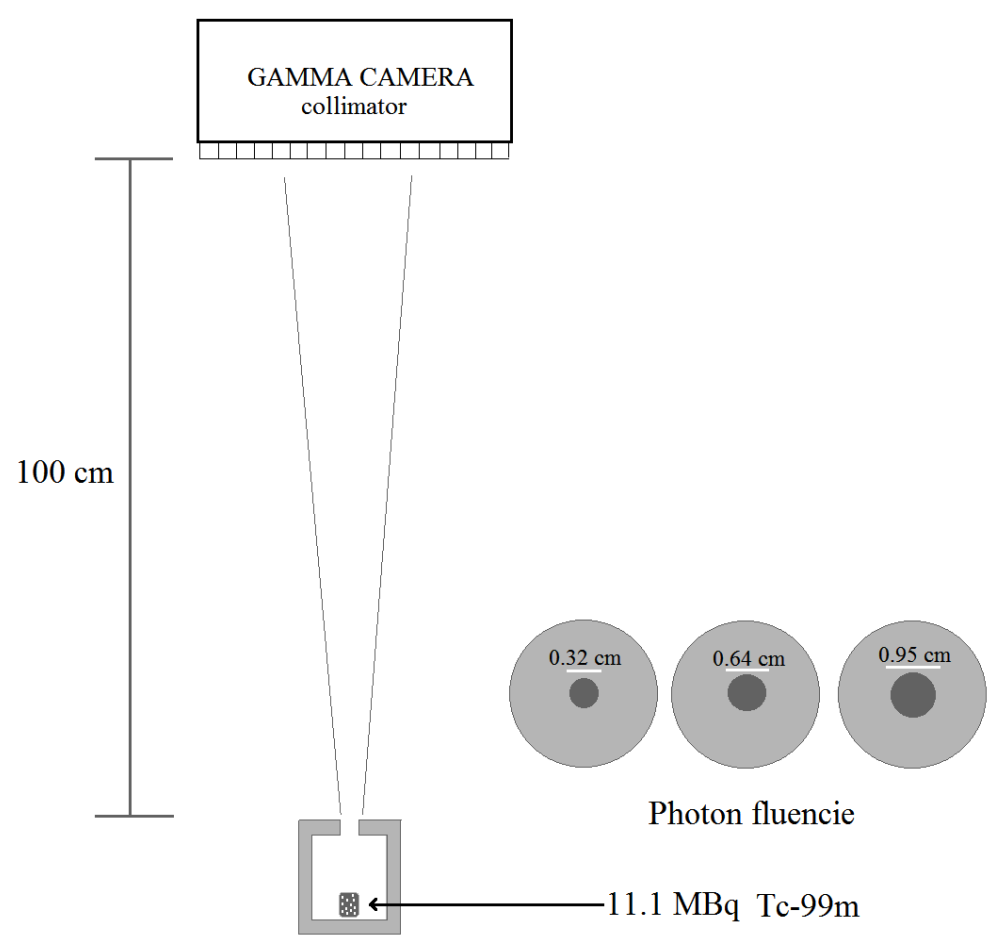

Figure 1. Experimental setup of narrowb eam geometry.

minutes the photons were counted for each measurement, three repetitions were performed for each thickness of agar gel and fluence. The number of counts recorded in the region of interest (ROI) was corrected only by background radiation, since in the narrow-beam geometry is assumed that no scattered radiation. The agar gel linear attenuation coefficient was determined by fitting the data of transmitted photons against thickness to an exponential function. The experimental setup was validated by measuring the water linear attenuation coefficient.

The XCOM database software provided by the NIST [24] was used to get an approximate value of agar gel linear attenuation coefficient for the concentrations (water:agar) used in this work. In the XCOM the chemical formula and mass fraction of agar powder and water used to prepare the gel samples were specified to calculate the mixture (agar gel) mass attenuation coefficient and it was divided by agar gel physical density to obtained linear attenuation coefficient.

The accuracy of agar gel linear attenuation coefficient $\mu_{\text {agar-gel }}$ obtained in this work was determined by Monte Carlo (MC) simulation, computing the photon transmission using this coefficient. The transmission data obtained by MC was compared with the results obtained experimentally. The MC code calculates the distance covered by the photon before its first interaction inside a defined thickness, using following expression [25]:

$$
s=-\frac{\ln r}{\mu_{\text {exp }}}
$$

where $r$ is a random number distributed between 0 and 1 .

As previously mentioned, a material is considered radiologically equivalent to water if it has the same effective atomic number, electron density and mass density [1]. Because of this, the mentioned parameters for agar gel were calculated in order to compare them with those of water.

The agar gel mass density was determined at room temperature by measuring the volume and weight of samples of agar gel using the following expression:

$$
\rho=\frac{m[\mathrm{~g}]}{v\left[\mathrm{~cm}^{3}\right]}
$$

The Mayneord equation was used to estimate the effective atomic number of agar powder, 


$$
Z_{\text {eff-agar }}=\sqrt[2.94]{\sum_{i=1}^{n} a_{i} z_{i}^{2.94}}
$$

where $a_{i}$ is the fractional contributions of the $i$-th element to the total number of electrons in the mixture and $z_{i}$ is the atomic number [1]. Finally, the agar gel effective atomic number was calculated with the following expression:

$$
Z_{\text {eff-gel }}=f_{a} Z_{\text {eff- } \mathrm{H}_{2} \mathrm{O}}+f_{b} Z_{\text {eff-agar }}
$$

where $f_{a}$ and $f_{b}$ represent the water and agar powder mass fraction used to prepare the agar gel samples.

The ${ }^{99 \mathrm{~m}} \mathrm{Tc}$ radiation dosimetry was performed using an agar gel cylindrical phantom with $4 \mathrm{~cm}$ diameter and $11.5 \mathrm{~cm}$ height, it was prepared with $18 \mathrm{MBq}$ of ${ }^{99 \mathrm{~m}} \mathrm{Tc}$ to simulate the source region. A scintigraphy image series acquired at various time obtained with conjugate view technique was used to estimate cumulated activity $\tilde{A}$ and the absorbed dose $(D)$ was estimated with the MIRD methodology. The source region was modeled with water and agar gel using the same geometry, volume and activity, to compare the activity $(A(t))$ and cumulated activity into both media. The source region was placed at $10 \mathrm{~cm}$ depth into a cylindrical water phantom with 24 $\mathrm{cm}$ diameter and $15 \mathrm{~cm}$ height and centered at its longitudinal axis. The Photopeak was centered at $140 \mathrm{keV}$. The activity at time $t$ was estimated using the Equation (5),

$$
A(t)=\sqrt{\frac{I_{A} I_{P}}{F}} \frac{f}{C}
$$

The $I_{A}$ and $I_{P}$ are the counts per minute of the anterior and posterior views corresponding to source region. The $I_{A}$ and $I_{P}$ were corrected by background [26] and scatter radiation using the dual energy window technique [27]. $C$ is the system calibration factor, it was obtained using the Equation (6),

$$
C=\left[\mathrm{cpm} / A_{C}\right]
$$

Where $A_{C}$ is the activity of a point source and $c p m$ are counts per minute registered in the gamma camera. $F$ is the transmission factor across the patient thickness, it was determined by Equation (7),

$$
F=\frac{I}{I_{0}}
$$

where $I$ and $I_{0}$ are counts registered with and without patient respectively. The experimental cumulated activity of water and agar gel phantom was represented by the area under the activity-time curve of the fitting function.

In two media the mean absorbed dose $(D)$ was estimated with the MIRD methodology, using the following equation:

$$
D=\tilde{A} * S_{t \leftarrow s}
$$

The $S$ value represents the absorbed dose per unit cumulated activity in the source region, and was determined with the following expression:

$$
S_{t \leftarrow s}=\frac{E_{i} n_{i} \phi_{t \leftarrow s}}{m_{\text {gel-phantom }}}
$$

where $E_{i}$ is the mean (or individual) energy emitted per nuclear transition, $n_{i}$ is number of its nuclear transitions per nuclear transformation and $\phi_{t \leftarrow s}$ is the fraction of energy emitted absorbed in the target region. $\phi_{t \leftarrow s}=1$ for low energy electrons emitted from ${ }^{99 \mathrm{~m}} \mathrm{Tc}$ and for its photons was estimated with:

$$
\phi_{t \leftarrow s} \simeq 1-e^{-\mu_{e n} \bar{r}}
$$

where $\mu_{e n}$ is the absorption coefficient and $\bar{r}$ is the mean radius of interest region [23].

\section{Results and Discussion}

The agar gel concentrations used in this study provided a solid medium which allow its easy manipulation. The $2.3 \%$ concentration produced a rigid gel and showed to be less brittle and less prone to fracture during its evalu- 
ation.

The results shown in Figure 2 indicate that the experimental setup of narrow beam geometry is valid and appropriate to obtain the linear attenuation coefficient for other materials, due to that the water linear attenuation coefficient value reported in the literature was obtained with this arrangement.

The calculated photon transmission through the agar gel is similar to that occurs in water as is shows in Figure 3 for the three fluencies, this is attributed to the chemical composition of agar gel which is almost completely composed by water [16], which suggests that the photon fraction removed from the radiation field is similar in both media.

The agar gel linear attenuation coefficients for both concentrations (agar:water) and the three beam diameters show differences among themselves about $5 \%$ (Table 1$)$, [ $\left.\bar{\mu}_{\text {agar-gel }}(1 \%)\right]$ is $0.5 \%$ less than $\left[\bar{\mu}_{\text {agar-gel }}(2.3 \%)\right]$. The mean linear attenuation coefficient of agar gel for the three fluencies was $\bar{\mu}_{\text {agar-gel }}=0.151 \mathrm{1} / \mathrm{cm}$, which is similar to the value in water with a difference of $0.7 \%$, when $\mu_{\text {water }}=0.151 / \mathrm{cm}$ [27], meanwhile there was no difference when the value is $\mu_{\text {water }}=0.1511 / \mathrm{cm}$ [23].

Figure 3 and Table 1 show that the accuracy and precision of the agar gel linear attenuation coefficient values obtained under these experimental conditions does not depend on photon fluencies, these differences and fluctuations can be related to the agar gel deformation [6], [28].

The theoretical verification of the experimental results was performed by XCOM program, where $\mu_{\mathrm{XCOM}, 1 \%}=$ $0.15061 / \mathrm{cm}$ and $\mu_{\text {Хсом, } 2.3 \%}=0.15071 / \mathrm{cm}$, comparing these values with that from Table $1\left(\bar{\mu}_{\text {agar-gel }}\right)$, a $0.5 \%$ difference was observed.

Using $\bar{\mu}_{\text {agar-gel }}$ the photon transmission was calculated by Monte Carlo simulation for both gels, the results in Figure 4 shows that the transmitted photon fraction is in agreement with that observed in the gamma camera with a maximum difference of $2 \%$.

The results suggest that the agar gel is an option for $140 \mathrm{keV}$ photon dosimetry, which compared to the materials from Table 2 [6], the agar gel has the advantage to be a material that allows geometry design, in addition to having a linear attenuation coefficient closest to that of the water.

Also the agar gel mass density was determined, where its mean value is $0.999 \pm 0.004 \mathrm{~g} / \mathrm{cm}^{3}$, it is approximately as that of water a $0.1 \%$ difference was observed.

To fully assess the radiological water equivalence of agar gel, the atomic number was determined. As mentioned in section methods, considering the fractional weight and chemical composition, the effective atomic number of agar gel as well as the corresponding value for water were obtained and show in Table 3 . As is well known, attenuation depends on the photon energy and effective atomic number, due to that from these parameters is define the interaction type [23], [28]. Moreover, the agar gel comparing with other gels, it shows greater similarity to water in terms of its radiological properties [8], [29].

The theoretical and experimental results of the photon transmission, linear attenuation coefficient and effective atomic number of the agar gel obtained in this work show that agar gel meets the ICRU (report 44) [30] requirements, thus the agar gel shown to be equivalent to water in terms of radiological properties for $140 \mathrm{keV}$ photons.

For dosimetry, the activity $A(t)$ was calculated by the conjugate view method. Using the Equations (6) and (7) and a point radiation source were determined $C=5091.7 \pm 261 \mathrm{cpm} / \mathrm{MBq}$ and $F=0.2247 \pm 0.0023$. A series of scintigraphic images were used to calculate the $A(t)$ as mentioned in methods section, the data are show in Figure 5, where source region was modeled with agar gel and water.

The $A^{\prime}(t)$ is the activity quantity at time $t$ by administered MBq to the source region. Based on the results shown in Figure 5, the $A^{\prime}(t)$ points were calculated in the two regions of interest (agar gel and water), by comparing the activity values corresponding at the same time, they show a difference less than $1 \%$. The last result is derived from the radiological equivalency between agar gel and water.

The cumulated activity was obtained by integrating the function $f(t)$, which is shown in Figure 5, is values is $\tilde{A}=124.4 \pm 1.7 \mathrm{MBq} * \mathrm{~h}$. On the other hand, by using the equations 9 and 10 were determined the absorbed dose per unit cumulated activity in the source region, namely $S=5.4 \times 10^{-4} \mathrm{~Gy} / \mathrm{MBq}$.

Finally, $D=0.06 \mathrm{~Gy} \pm 0.002$ was the absorbed dose calculated for two phantoms.

The radiological water equivalence was assessed by comparing the densities, atomic number and radiation 


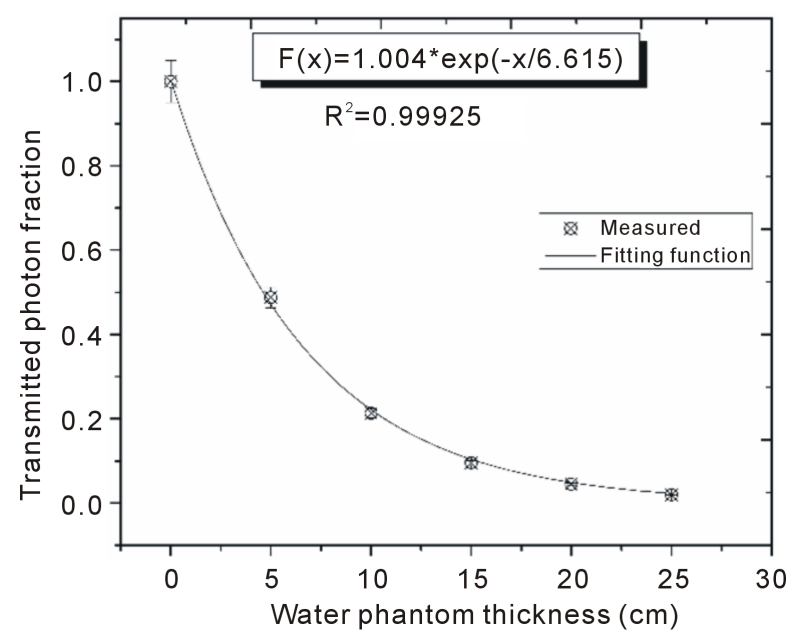

Figure 2. $140 \mathrm{keV}$ photon transmission through water using narrow beam geometry.
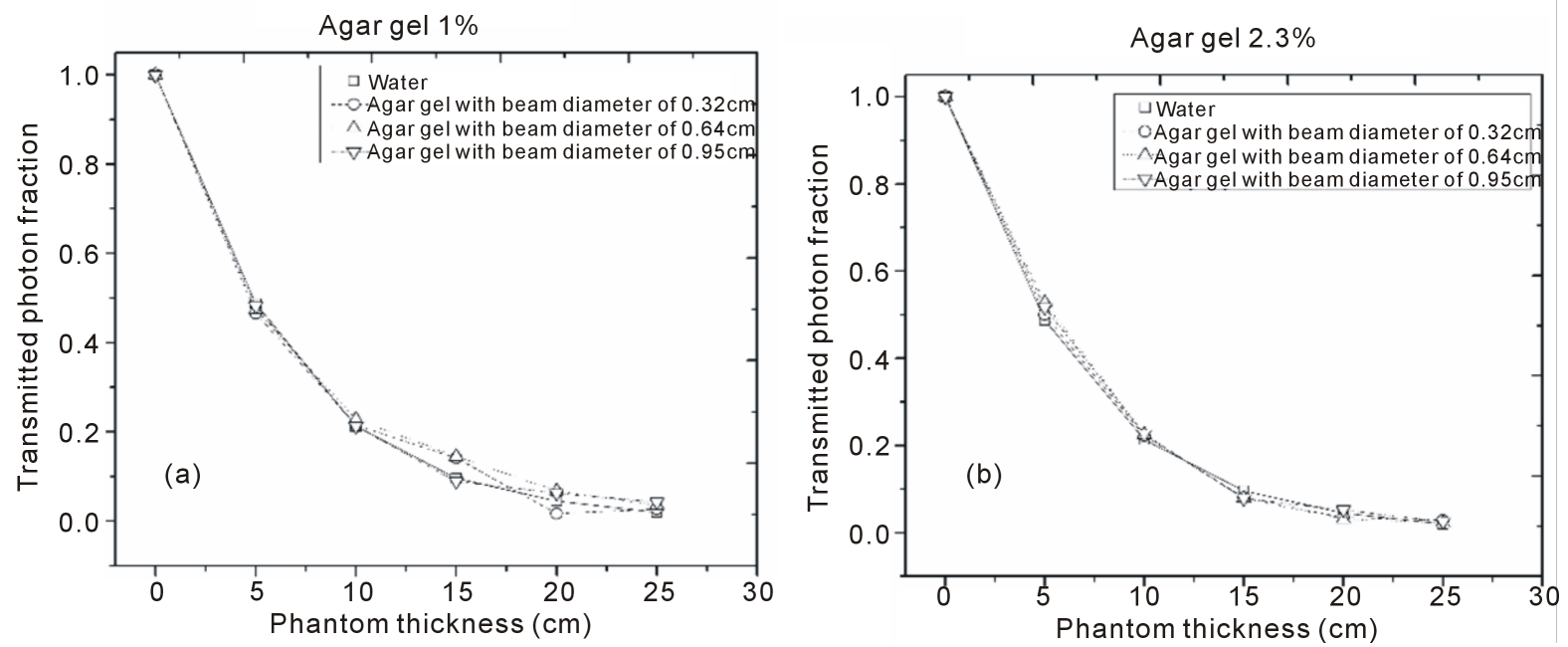

Figure 3. Transmission values of technetium-99m gamma rays passing through water and agar gel. (a) Agar gel 1\%, (b) Agar gel 2.3\%.
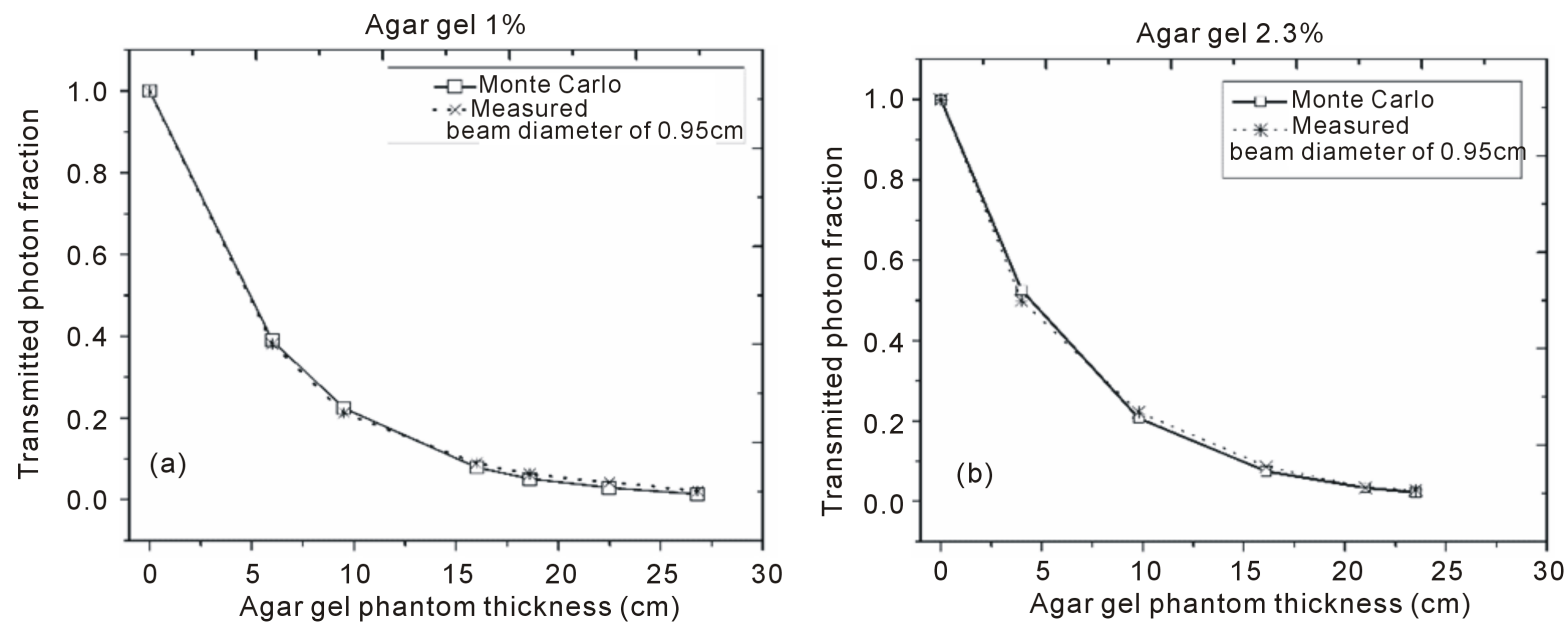

Figure 4. $140 \mathrm{keV}$ photons transmission, experimental and Monte Carlo results. (a) Agar gel 1\%, (b) Agar gel 2.3\%. 
Table 1. Agar gel linear attenuation coefficient.

\begin{tabular}{cccc}
\hline Beam diameter $[\mathrm{cm}]$ & Fitting function & $\mu_{\text {agar gel } \_1 \%\left[\mathrm{~cm}^{-1}\right]}$ & $\pm \sigma_{1}$ \\
\hline 0.32 & $f(x)=0.99 * \exp (-x / 6.79)$ & 0.148 & 0.017 \\
0.64 & $f(x)=0.99 * \exp (-x / 6.88)$ & 0.146 & 0.013 \\
0.95 & $f(x)=0.997 * \exp (-x / 6.34)$ & 0.158 & 0.004 \\
$\mu_{\text {agar gel }}$ & Fitting function & 0.1506 & 0.004 \\
& $f(x)=1.01 * \exp (-x / 6.88)$ & $\mu_{\text {agar gel }} 2.3 \%\left[\mathrm{~cm}^{-1}\right]$ & $\pm \sigma_{2}$ \\
0.32 & $f(x)=1.01 * \exp (-x / 6.69)$ & 0.145 & 0.005 \\
0.64 & $f(x)=0.99 * \exp (-x / 6.22)$ & 0.149 & 0.005 \\
0.95 & & 0.160 & 0.007 \\
$\mu_{\text {agar gel }}$ & & 0.1513 & 0.002 \\
\hline
\end{tabular}

Table 2. Linear attenuation coefficients, $\mu(1 / \mathrm{cm})$, obtained from photon transmission and calculated by XCOM program for $140 \mathrm{keV}$ photons emitted by a ${ }^{99 \mathrm{~m}}$ Tc source.

\begin{tabular}{|c|c|c|c|c|}
\hline & Agar gel & RMI-457 & Plastic water & RW3 \\
\hline$\mu_{\text {experimental }}\left(\mathrm{cm}^{-1}\right)$ & 0.151 & 0.151 & 0.151 & 0.149 \\
\hline$\mu_{X C O M}\left(\mathrm{~cm}^{-1}\right)$ & 0.151 & 0.154 & 0.155 & 0.155 \\
\hline
\end{tabular}

Table 3. Agar gel and water effective atomic number ('Khan, 2010).

\begin{tabular}{ccc}
\hline Material & $Z_{\text {eff-gel }}$ \\
\hline Agar gel, 1\% & 7.50 \\
Agar gel, 2.3\% & 7.50 & 7.51 \\
\hline
\end{tabular}

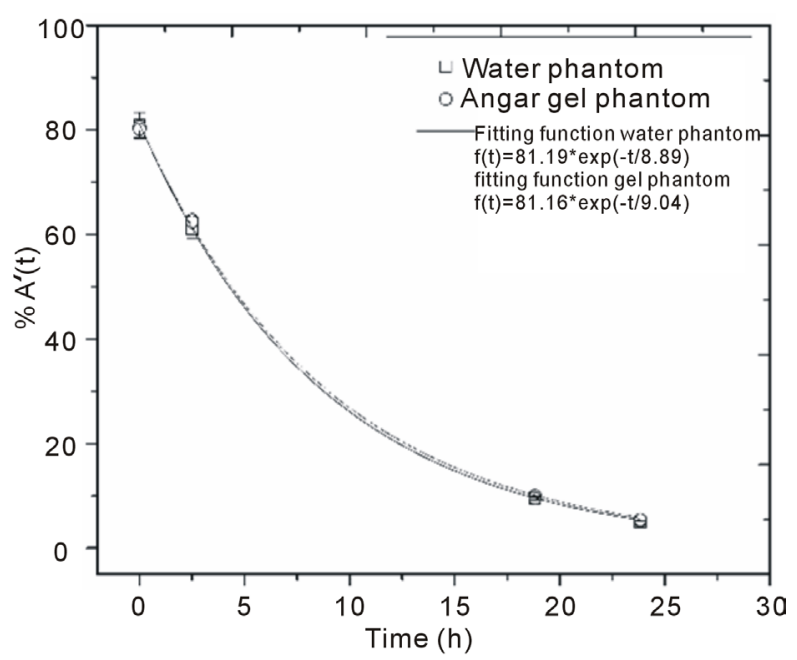

Figure 5. $A^{\prime}(t)$ in source region modeled with agar gel and water.

dosimetry properties of the agar gel. Thus, this gel can be used with radiometric purpose as soft biological tissue substitute when it is irradiated with $140 \mathrm{keV}$ photons.

\section{Conclusions}

The agar gel prepared at a concentration of $2.3 \%$ showed to be a stable material with adequate mechanical strength for its handling, capable of reproduce three-dimensional shapes that can model different organs or biological tissue.

It has been shown that the gamma camera with all its limitations in terms of energy discrimination of photons 
registered under narrow beam geometric conditions is a good choice to measure the linear attenuation coefficient of materials irradiated with gamma rays from ${ }^{99 \mathrm{~m}} \mathrm{Tc}$.

The agar gel is a medium that can replace water because it has almost the same linear attenuation coefficient $\bar{\mu}_{\text {agar-gel }}=0.151 \mathrm{1} / \mathrm{cm}$ when this medium is irradiated with $140 \mathrm{keV}$ photons.

In accordance with ICRU-report 44, the agar gel presented radiological properties as density, effective atomic number and linear attenuation coefficient for a radiation field of $140 \mathrm{keV}$ photons showing the radiological water equivalence.

Based on the literature results and results from this work, the agar gel is a medium that can substitute biological tissue for the radiometric and optical characterization of pharmaceuticals containing gold nanoparticles and radionuclides.

\section{Acknowledgements}

This work was supported by the Universidad Autónomadel Estado de México (3227/2012CHT, 3416/2013CHT), Department of Nuclear Medicine, Centro Oncológico del Estado de México for allowing the use of equipment and providing the technetium-99m radionuclide.

\section{References}

[1] Khan, F.M. (2010) The Physics of Radiation Therapy. 4th Edition, Lippincott Williams \& Wilkins, Philadelphia.

[2] White, D.R. (1978) Tissue Substitutes in Experimental Radiation Physics. Medical Physics, 5, 467-480. http://dx.doi.org/10.1118/1.594456

[3] Keall, P., Kron, T. and Hoban, P. (1993) A Monte Carlo Technique to Establish the Water/Tissue Equivalence of Phantom Materials. Australasian Physical and Engineering Science in Medicine, 16, 125-128.

[4] Pantelis, E., Karlis, A.K., Kozicki, M., Papagiannis, P., Sakelliou, L. and Rosiak, J.M. (2004) Polymer Gel Water Equivalence and Relative Energy Response with Emphasis on Low Photon Energy Dosimetry in Brachytherapy. Physics in Medicine and Biology, 49, 3495-3514. http://dx.doi.org/10.1088/0031-9155/49/15/013

[5] Sellakumar, P., James, J.S. and Supe, S. (2007) Water Equivalence of Polymer Gel Dosimeters. Radiation Physics and Chemistry, 76, 1108-1115. http://dx.doi.org/10.1016/j.radphyschem.2007.03.003

[6] Hill, R., Brown, S. and Baldock, C. (2008) Evaluation of the Water Equivalence of Solid Phantoms Using Gamma Ray Transmission Measurements. Radiation Measurements, 43, 1258-1264. http://dx.doi.org/10.1016/j.radmeas.2008.01.019

[7] Midgley, S.M. (2005) Measurements of the X-Ray Linear Attenuation Coefficient for Low Atomic Number Materials at Energies 32-66 and $140 \mathrm{keV}$. Radiation Physics and Chemistry, 72, 525-535. http://dx.doi.org/10.1016/j.radphyschem.2004.02.001

[8] Gorjiara, T., Hill, R., Kuncic, Z., Bosi, S., Davies, J. and Baldock, C. (2011) Radiological Characterization and Water Equivalency of Genipin Gel for X-Ray and Electron Beam Dosimetry. Physics in Medicine and Biology, 56, 4685-4699. http://dx.doi.org/10.1088/0031-9155/56/15/004

[9] Traub, R.J., Olsen, P.C. and Mcdonald, J.C. (2006) The Radiological Properties of a Novel Lung Tissue Substitute. Radiation Protection Dosimetry, 121, 202-207. http://dx.doi.org/10.1093/rpd/nci371

[10] Venning, A.J., Nitschke, K.N., Keall, P.J. and Baldock, C. (2005) Radiological Properties of Normoxic Polymer Gel Dosimeters. Medical Physics, 32, 1047-1053. http://dx.doi.org/10.1118/1.1881812

[11] Taylor, M.L., Franich, R.D., Trapp, J.V. and Johnston, P.N. (2008) The Effective Atomic Number of Dosimetric Gels. Australasian Physical and Engineering Sciences in Medicine, 31, 131-138. http://dx.doi.org/10.1007/BF03178587

[12] Constantinou, C. (1982) Phantom Materials for Radiation Dosimetry. I. Liquids and Gels. British Journal of Radiology, 55, 217-224. http://dx.doi.org/10.1259/0007-1285-55-651-217

[13] Hartmann-Siantar, C.L., Walling, R.S., Daly, T.P., Faddegon, B., Albright, N., Bergstrom, P., Bielajew, A.F., Chuang, C., Garrett, D., House, R.K., Knapp, D., Wieczorek, D.J. and Verhey, L.J. (2001) Description and Dosimetric Verification of the PEREGRINE Monte Carlo Dose Calculation System for Photon Beams Incident on a Water Phantom. Medical Physics, 28, 1322-1337. http://dx.doi.org/10.1118/1.1381551

[14] Cubeddu, R., Pifferi, A., Taroni, P., Torricelli, A. and Valentini, G. (1997) A Solid Tissue Phantom for Photon Migration Studies. Physics in Medicine and Biology, 42, 1971-1979. http://dx.doi.org/10.1088/0031-9155/42/10/011

[15] Romo, G. and Camacho, S. (2007) Efectos de Calentamiento y Formación de Burbuja Inducidos con Láseres Pulsados en Modelos de Tejido-Biológico. Tesis de Maestría. Centro de Investigación Científica y de Educación Superior de 
Ensenada.

[16] Escobar, J.L., García, D.M., Zaldivar, D. and Katime, I. (2002) Hidrogeles: Principales Características en el Diseño de Sistemas de Liberación Controlada de Fármacos. Revista Iberoamericana Polímeros, 3, 1-25.

[17] Pal, K., Banthia, A.K. and Majumdar, D.K. (2009) Polymeric Hydrogels: Characterization and Biomedical Applications: A Mini Review. Designed Monomers and Polymers, 12, 197-220. http://dx.doi.org/10.1163/156855509X436030

[18] Milanič, M., Majaron, B. and Stuart, J. (2007) Pulsed Photothermal Temperature Profiling of Agar Tissue Phantoms. Lasers in Medical Science, 22, 279-284. http://dx.doi.org/10.1007/s10103-007-0455-9

[19] Melancon, M., Lu, W., Yang, Z., Zhang, Z., Cheng, Z., Stafford, J., Olson, T., Zhang, J. and Li, C. (2008) In Vitro and in Vivo Targeting of Hollow Gold Nanoshells Directed at Epidermal Growth Factor Receptor for Photothermal Ablation Therapy. Molecular Cancer Therapy, 7, 1730-1739. http://dx.doi.org/10.1158/1535-7163.MCT-08-0016

[20] Jiménez, N., Ferro, G., Ocampo, B., Luna, M., Ramírez, F., Pedraza, M. and Torres, E. (2012) Multifunctional Targeted Radiotherapy System for Induced Tumours Expressing Gastrin-Releasing Peptide Receptors. Current Nanoscience, 8, 193-201. http://dx.doi.org/10.2174/157341312800167678

[21] Ocampo, B., Ferro, G., Morales, E. and Ramírez, F. (2011) Kit for Preparation of Multimeric Receptor Specific 99mTc Radiopharmaceuticals Based on Gold Nanoparticles. Nuclear Medicine Communications, 32, 1095-1104. http://dx.doi.org/10.1097/MNM.0b013e32834acf33

[22] http://www.chemblink.com/products/9002-18-0.htm

[23] Attix, F.H. (1986) Introduction to Radiological Physics and Radiation Dosimetry. J. Wiley and Son, New York. http://dx.doi.org/10.1002/9783527617135

[24] http://physics.nist.gov/PhysRefData/Xcom/Text/XCOM.html

[25] Snyder, W., Ford, M. and Warner, G. (1978) MIRD Pamphlet No. 5. Revised: Estimated of Specific Absorbed Fractions for Photon Sources Uniformly Distributed in Various Organs of a Heterogeneous phantom.

[26] Buijs, W., Siegel, J.A., Boerman, O. and Corstens, F. (1998) Absolute Organ Activity Estimated by Different Methods of Background Correction. Journal of Nuclear Medicine, 39, 2167-2172.

[27] Siegel, J.A., Thomas, S.R., Stubbs, J.B. and Stabin, M.G. (1999) MIRD Pamphlet No. 16: Techniques for Quantitative Radiopharmaceutical Biodistribution Data Acquisition and Analysis for Use in Human Radiation Dose Estimates. Journal of Nuclear Medicine, 40, 31-68.

[28] Hill, L., Holloway, L. and Baldock, C. (2005) A Dosimetric Evaluation of Water Equivalent Phantoms for Kilovoltage X-Ray Beams. Physics in Medicine and Biology, 50, 331-344. http://dx.doi.org/10.1088/0031-9155/50/21/N06

[29] Brown, S., Venning, S., De Deene, Y., Vial, P., Oliver, L., Adamovics, J. and Baldock, C. (2008) Radiological Properties of the PRESAGE and PAGAT Polymer dosimeters. Applied Radiation and Isotopes, 66, 1970-1974. http://dx.doi.org/10.1016/j.apradiso.2008.06.005

[30] ICRU (1989) Tissue Substitutes in Radiation Dosimetry and Measurement (Report 44). 